\title{
ASCCP guidelines for managing abnormal cervical cancer tests: What's new?
}

\author{
They've traded in algorithms for risk, and there will soon be a \\ new app to streamline navigation of the guidelines
}

\author{
Q\&A with Warner K. Huh, MD
}

$\mathrm{T}$ he 2019 ASCCP Risk-Based Management Consensus Guidelines for Abnormal Cervical Cancer Screening Tests and Cancer Precursors Consensus Guidelines, which represent a consensus of nearly 20 professional organizations and patient advocates, are a culmination of almost 10 years of research. ${ }^{1}$ With the last version issued in $2012{ }^{2}$ these latest guidelines offer the most recent recommendations regarding safely triaging women with abnormal cervical cancer screening results.

According to the consensus, research has shown that risk-based management allows clinicians to better discriminate women who will likely develop precancer from those who can safely continue with routine screening. As you will hear from guidelines coauthor Dr. Warner Huh, one of the most important differences between these guidelines and the 2012 version is a new emphasis on the principle of "equal

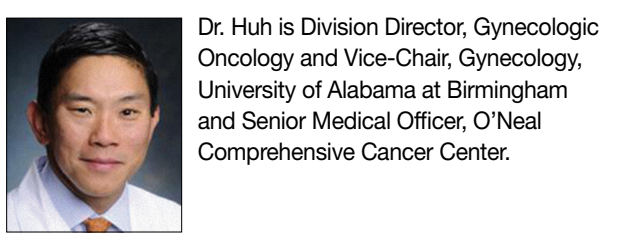

The author reports being a consultant to Inovio, Zilico, and Altum. management for equal risk." Essentially, this insures that all women who have the same amount of risk for progression to precancer or cancer are managed the same.

The guidelines were once again published in the Journal of Lower Genital Tract Disease, and the tables they reference are publicly available. Additionally, ASCCP is developing a new management guidelines app to facilitate the use of the guidelines on smartphones and computers. With the publicly available risk tables, and the ASCCP navigation app, the guidelines will more easily accommodate updates as new information and technology become available.

OBG MANAGEMENT: The latest ASCCP guidelines, published in April, represent a "paradigm shift" from results to risk-based guidelines. Can you explain what this means and why the shift was undertaken? Warner K. Huh, MD: Yes, the shift occurred because we needed to focus less on algorithms and more on risk. We started promulgating a concept of "equal management for equal risk" back in 2012. What this means is that if we have a method to look up a risk score based on relevant test results and other pieces of information, then all patients with that score should be managed in the same
IN THIS ARTICLE

App availability page 44

Changes from prior guidelines

page 46 
$\overline{\text { FAST }}$

TRACK

Risk-based

guidelines have

the advantages

of being easier

to use, incorporating

new data efficiently

and effectively,

and allowing

for future

technologies manner. We wanted that to be the underlying principle.

Focusing on risk tables also makes it easier to incorporate any future technologies used for risk estimation without having to rebuild algorithms from scratch. ASCCP is developing a new management guidelines app to streamline navigation of the guidelines. This app makes them easier for clinicians to use; they simply plug in certain variables from the patient's history and receive 1 of 5 outputs: treatment, colposcopy, or surveillance at 1,3 , or 5 years.

The only drawbacks, if you view them as such, are that the clinician must plug in all the variables, and then must sit back and trust in what we have done. Clinicians have to trust that the system works and will simplify the clinical decision making.

We spent a lot of time determining what the risk thresholds should be. Some may argue they are arbitrary, but the decisions were datadriven, and carefully, thoughtfully vetted; we deliberated about whether the cut points actually made sense clinically to a practicing clinician base. The clinical action thresholds refer to a specific percentage below which a woman falls into one bucket and above which she falls into another bucket.

The other element that is unique about the guidelines is that instead of looking at the patient's current screening result in isolation, the user sees it along with the prior one because prior history dictates subsequent risk.

It's important that clinicians understand why this system is so markedly different from what we have done previously, and why riskbased guidelines make infinitely more sense than algorithmic ones. It's because: 1) they can be easier to use; 2) they incorporate new data more efficiently and effectively than algorithm-based guidelines; and 3) they can incorporate future technologies seamlessly rather than having to create yet another algorithm.

\section{OBG MANAGEMENT: What do clinicians need in order to execute the guidelines?}

Dr. Huh: Nothing. All of the information needed-the guidelines article and risk tables-are publicly available. However, to make navigation of the guidelines easier, the plan is for the app that I mentioned. I have the app on my phone and am actively beta testing it now. We're planning on creating a web-based application as well, that will allow users to access the Internet and their electronic health record system so that they can plug in information directly from patient charts. The web-based app will be similar to the web-based Breast Cancer Surveillance Consortium's Risk Calculator (https://tools .bcsc-scc.org/BC5yearRisk/calculator.htm). You will pull it up, plug in the requested information, including the patient's age; their Pap smear and genotyping results; and their previous screening history.

\section{OBG MANAGEMENT: When will the app be available for users?}

Dr. Huh: It will be available for release on June 8.

\section{OBG MANAGEMENT: Were HPV} vaccination levels incorporated into the new guidelines?

Dr. Huh: We initially looked at them because human papillomavirus (HPV) vaccination hugely influences outcomes but, no, we did not include them in the guidelines. The reason is that it's really challenging to prove whether a woman has been vaccinated. You have to have access to vaccine records. Then there is also the issue of whether a patient has had 1,2, or 3 doses. That is a really sticky variable. So, since it is not part of the guidelines, ASCCP also did not include it as a part of the app or the website. But we do recognize that HPV vaccination plays an important role in outcomes.

\section{OBg Management: Have recommendations regarding colposcopy changed?}

Dr. Huh: Not really. About 3 years ago, we created basic colposcopy guidelines-the ASCCP Colposcopy Standards-so everything about colposcopy references back to those guidelines. Those colposcopy standards covered terminology and risk-based 
colposcopy, which actually aligns beautifully with these guidelines.

\section{OBG MANAGEMENT: To narrow in on some changes from the prior guidelines, can colposcopy be deferred in certain patients?}

Dr. Huh: Yes. Not everyone who has an abnormal screening test needs to come back for colposcopy.

\section{OBG MANAGEMENT: How has guidance for expedited treatment or treatment without colposcopic biopsy changed?}

Dr. Huh: This was heavily debated within not only the treatment group that I co-chaired with Richard Guido, MD, but also within the entire steering committee. The recommendation is that if the patient has an immediate risk of CIN 3 that is $>60 \%$, the patient should go straight to treatment without a colposcopic biopsy. The main reason for this is that you do not want to biopsy a patient and then lose them to follow-up.

References

1. Perkins RB, Guido RS, Castle PE, et al, for the 2019 ASCCP RiskBased Management Consensus Guidelines Committee. 2019 ASCCP Risk-Based Management Consensus Guidelines for Abnormal Cervical Cancer Screening Tests and Cancer Precursors. J Low Genit Tract Dis. 2020;24(2):102-131.
When a woman has $>60 \%$ immediate risk of CIN 3, we are fairly certain that colposcopy is not going to change management ultimately, so we recommend that patients receive treatment right away. We have already been doing this for 15 to 20 years, so this is not a new concept. It is just more formally codified here by assigning a percentage to the risk. Those who have between $25 \%$ and $60 \%$ immediate risk of CIN 3 should receive immediate colposcopy. We realize that not all clinicians have the ability to do this, so if clinicians can't treat immediately, we recommend they do whatever they can to prevent losing the patient to follow-up.

\section{OBG MANAGEMENT: How should a positive primary HPV screening test be managed?}

Dr. Huh: If a woman has a positive primary HPV screening test, genotyping should be performed. If genotyping reveals HPV 16 or 18 , then the patient should proceed to colposcopy. If genotyping reveals other forms of HPV, reflex cytology or a Pap smear should follow.

2. Massad LS, Einstein MH, Huh WK, et al, for the 2012 ASCCP Consensus Guidelines Conference. 2012 Updated Consensus Guidelines for the Management of Abnormal Cervical Cancer Screening Tests and Cancer Precursors. J Low Genit Tract Dis. 2013;17(5):S1-S27. 